\title{
Application of polarization interferometers for Thomson scattering
}

\author{
J Howard \\ Plasma Research Laboratory, Australian National University, Canberra, ACT 0200, Australia \\ E-mail: john.howard@anu.edu.au
}

Received 11 October 2005, in final form 9 March 2006

Published 28 April 2006

Online at stacks.iop.org/PPCF/48/777

\begin{abstract}
Wide field-of-view, high transparency birefringent filters (essentially fixed delay interferometers) are proposed for incoherent Thomson scattering measurements of the temperature and density of plasma free electrons. For thermal electrons, the optical coherence of the Thomson scattered light at an appropriately chosen optical path delay, is a unique function of the electron temperature and density. The detection system utilizes a single filter combined with imaging optics and dual detector arrays to simultaneously observe both dark and bright scattered light interference fringes. The system delivers two signals that allow the recovery of the two unknowns - temperature and density. It is shown that the normalized intensity difference between the bright and dark interference fringes gives a direct measure of the electron temperature, even for strongly blue shifted high temperature spectra. As usual, the total scattered light flux (the sum of bright and dark signals) is proportional to the number of illuminated electrons. For multi-pulse systems, an electronically switchable ferroelectric liquid crystal delay plate synchronized with the laser repetition rate can allow density and temperature to be obtained using a single detector array. The use of a time-multiplex approach both simplifies relative channel calibration issues and opens the possibility for $2 \mathrm{D}$ temperature imaging. This paper describes the measurement principle and presents the results of numerical simulations for both low and high temperature scenarios.
\end{abstract}

(Some figures in this article are in colour only in the electronic version)

\section{Introduction}

Laser Thomson scattering from free plasma electrons [1] is now the standard technique for measuring electron temperature in almost all terrestrial plasmas, from low temperature atmospheric arcs, through to magnetic fusion devices. Because of their small mass, the electrons can have velocities that are a substantial fraction of the speed of light. As a result 
the light scattered from an illuminating high-power pulsed laser source has high spectral bandwidth (low coherence). The scattered spectrum is usually measured using a low-resolution high-throughput grating spectrometer. By equipping the spectrometer with a 2-D intensified and gated CCD camera, it is possible to image the entire laser stripe in the plasma and so obtain spatially resolved electron temperature and density information, albeit at a relatively low repetition rate. [2].

A common approach to obtaining time resolved scattered spectra in multi-pulse laser systems is to use interference filter polychromators combined with an array of photomultipler tubes or avalanche phototdiode detectors. By carefully calibrating the filters it is possible to use the measured intensity ratios to estimate the spectral width and hence the electron temperature. Though imaging filter polychromators and detector arrays have been used successfully [3,4], more often, each spatial channel uses a dedicated polychromator with a photo-detector for each spectral bin. For high spatial resolution systems, this presents a formidable problem in terms of expense, maintenance and calibration.

We propose a method based on measurement of the optical coherence of the scattered radiation at a fixed optical delay. High-throughput, wide field-of-view polarization interferometers have been used recently for Doppler imaging of ion temperature in the $\mathrm{H}-1$ heliac [5-7]. These modulated or static coherence imaging systems monitor the complex coherence (fringe visibility and phase) of an isolated spectral line at one or more optical delays. When observing ion motions, the spectral width is small compared with the the light wavelength, necessitating the use of high spectral resolution (large delay) optical interferometers. In this article, we focus on the utility of birefringent plate interferometers for relatively low resolution spectral applications such as Thomson scattering. For convenience, we shall also refer to these birefringent interferometers as filters in the sense that it is their time-domain response that is narrowband (single delay) as opposed to their optical frequency domain response which is oscillatory.

In its simplest form, the collected scattered light is transmitted by a wide passband interference pre-filter to a polarization interferometer that generates an image of the optical coherence at some pre-selected optical path length delay. The polarization interferometer is essentially a birefringent optical delay plate sandwiched between either parallel or crossed polarizers. The delay plate fast axis is at $45^{\circ}$ to the first polarizer axis. Depending on the orientation of the final polarizer, it is possible to measure either a 'bright' or 'dark' interference fringe as explained in section 2 below. By using a Wollaston prism as the final polarizing element, distinct images of the antiphase components of the optical coherence (bright and dark fringes) can be formed onto parallel detector arrays. The difference between the bright and dark fringe intensities $S_{+}$and $S_{-}$normalized to the mean bears a simple monotonic relationship to the electron temperature, while the mean of the two signals normalized to the pulse energy is proportional to the electron density.

In practice, the received signals will be the summation of the interferograms for the Thomson scattered and stray laser light as well as plasma background emission. In common with other Thomson systems, the background component can be independently measured in a nearby time window and its interferogram subsequently subtracted. Stray light has long coherence length. Its contribution can be subtracted based on signals obtained in the absence of plasma, or by monitoring the interferogram fringe visibility at delays greater than the coherence length of the Thomson scattered spectrum.

In a sense, our approach is equivalent to forming the normalized difference of scattered intensities received in adjacent passbands transmitted by independent interference filters. However, as we show here, a single birefringent interferometer is capable of resolving temperatures over a very wide range. By virtue of its high throughput, it is especially 
well suited to the measurement of very low temperatures $(\sim 1 \mathrm{eV})$ which require moderate spectral resolving power. Birefringent filters also offer some advantages in terms of alignment simplicity and wide field-of-view and as such, are readily amenable to imaging applications. For example, because of the simple relationship between fringe contrast and electron temperature, 2D temperature imaging becomes feasible using a CCD camera to capture the angularly displaced antiphase images. The plasma is illuminated using either a high power expanded laser beam, or by translating a multiply-reflected laser beam across the plasma region of interest.

As an alternative to measuring simultaneously both polarization components, temporal multiplex techniques based on electro-optically switchable waveplates can be of advantage in repetitive-pulse or modulated continuous-wave laser Thomson systems (see for example [8]). We propose the use of ferro-electric liquid crystals for which the orientation of the crystal fast axis can be electronically controlled. By synchronizing waveplate switching with the laser pulse rate it is possible to combine signals from alternate pulses to obtain electron density and temperature using a single detector element per spatial channel. This has the advantage of eliminating the need for relative channel spectral sensitivity calibration. Conceivably, such systems could also be configured to improve the sensitivity of the temperature measurement by using multiple switchable delays to access different regions of the optical coherence.

This paper is organized as follows. In section 2 we briefly review the relationship between the Thomson scattered spectrum and its optical coherence. We then describe a birefringent interferometer suitable for simultaneously monitoring orthogonally polarized output images of light scattered from a probing laser beam. The filter response, including material dispersive properties, is modelled for both low and high temperature cases. We also present a timemultiplex variant of the split-image system suitable for use in repetitive-pulse systems. This approach has the advantage of requiring only a single detector element per spatial channel. In section 3 we discuss experimental considerations as they apply to the use of birefringent filters, namely stray and plasma light issues, and suggest a means of calibration of the system response in the difficult, high-temperature, wide passband case.

\section{Measurement principle}

The polarization interferometer is composed of a birefringent plate of fixed or switchable optical delay sandwiched between polarizers. Scattered radiation that traverses the first polarizer is incident on a birefringent plate whose fast axis is oriented at $45^{\circ}$ to the polarization direction. The plate splits the incident scattered scalar wave component $u(t)$, relatively delaying nominally equal amplitude components by time $\tau$ before they are recombined at a final polarizer and focused onto a square-law detector. The orthogonally polarized outputs at the final polarizing splitter form complementary, or antiphase interferometric images of the input radiation. By suitably choosing the optical delay, these independent outputs provide sufficient information to determine both the electron density and temperature.

The intensity of the transmitted or reflected light is proportional to

$$
S_{ \pm}(\tau)=\frac{I_{0}}{2}[1 \pm \Re[\tilde{\gamma}(\tau)]],
$$

where $I_{0}=\left\langle u u^{*}\right\rangle$ is the spectrally integrated irradiance, or brightness, and $\tilde{\gamma}$ is the complex coherence given by $\tilde{\gamma}=\left\langle u(t) u^{*}(t+\tau)\right\rangle / I_{0}$ where angle brackets denote a time average [9]. In general, the complex temporal coherence is related to the spectral distribution of the irradiance 
$I(v)$ through the Wiener-Khinchine theorem

$$
\tilde{\gamma}(\tau)=\frac{1}{I_{0}} \int_{-\infty}^{\infty} I(\nu) \exp (12 \pi \nu \tau) d \nu .
$$

For relatively narrowband spectra, the interferometric phase delay can be approximated by

$$
\begin{aligned}
\phi & =2 \pi \nu \tau(\nu) \\
& \approx \phi_{0}+\kappa \phi_{0} \xi,
\end{aligned}
$$

where we have substituted $v=v_{0}(1+\xi)$, where $\xi=\left(v-v_{0}\right) / v_{0}$ is a normalized frequency difference coordinate, $\phi_{0}=2 \pi v_{0} \tau_{0}$, where $\tau_{0}$ is the centre-frequency time delay and where

$$
\kappa=1+\left.\frac{\nu_{0}}{\tau_{0}} \frac{\partial \tau}{\partial v}\right|_{\nu_{0}}
$$

accounts for any optical frequency dispersion of the time delay. Using (3) in equations (1) and (2) obtains the interferogram

$$
S_{ \pm}\left(\phi_{0}\right)=\frac{I_{0}}{2}\left[1 \pm \Re\left[\tilde{\gamma}\left(\hat{\phi}_{0}\right) \exp \left(1 \phi_{0}\right)\right]\right]
$$

where the optical coherence is given by

$$
\tilde{\gamma}(\hat{\phi})=\frac{1}{I_{0}} \int_{-\infty}^{\infty} I(\xi) \exp (i \hat{\phi} \xi) d \xi
$$

and it is convenient to introduce the group phase delay

$$
\hat{\phi}_{0}=\kappa \phi_{0} \text {. }
$$

For more general or broadband spectra, equation (2) must be evaluated numerically.

For a given scattering geometry, and assuming the electrons to be in thermal equilibrium, the shape of the incoherent Thomson spectrum can be generally expressed in terms of of the electron temperature alone. Accurate analytic approximations to the form of the spectrum valid for relativistic energies have been reported [10]. The scattered spectrum gives information about the velocity distribution function in the direction $\boldsymbol{k}=\boldsymbol{k}_{s}-\boldsymbol{k}_{i}$, where $\boldsymbol{k}_{s}$ and $\boldsymbol{k}_{i}$ are wavevectors for the scattered and incident beams, respectively [11]. We can evaluate the polarization interferometer response analytically in the low temperature case where the scattered spectrum is approximately Gaussian. For the high temperature case, the interferogram is calculated numerically taking into account optical-frequency-dependent material parameters.

\subsection{Low temperature case}

For low electron temperatures (less than a few hundred electronvolts), the spectral dependence of the scattered radiation is well approximated by

$$
I_{T}\left(\xi ; T_{\mathrm{e}}\right)=I_{0}\left(\pi \xi_{\mathrm{th}}^{2}\right)^{-1 / 2} \exp \left(-\xi^{2} / \xi_{\mathrm{th}}^{2}\right)
$$

with

$$
\xi_{\text {th }} \equiv 2 \sin (\theta / 2) v_{\text {th }},
$$

and where $\theta$ is the angle between the incident and scattered wavevectors, $T_{\mathrm{e}}$ is the electron temperature and $v_{t h}$ is the electron thermal speed normalized to the speed of light. Because the spectrum is a function of only a single parameter, measurement of the coherence at a single fixed delay offset $\phi_{0}$ is sufficient to obtain $T_{\mathrm{e}}$.

The simple birefringent filter shown schematically in figure 1, uses interference techniques to estimate the optical coherence length. The normal-incidence time delay introduced by a 


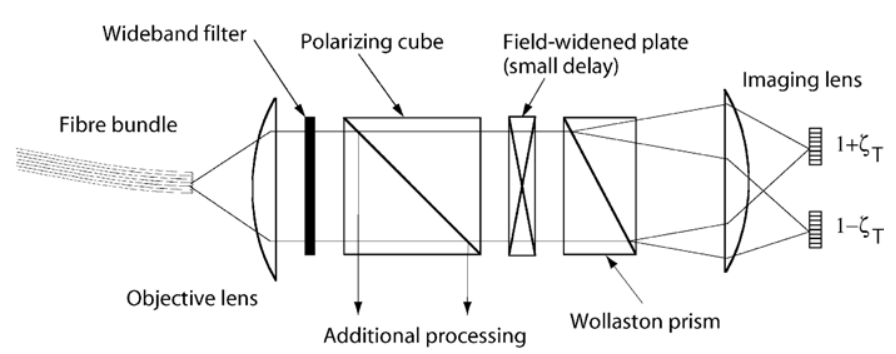

Figure 1. Layout for a simple coherence imaging filter for Thomson scattering. The final Wollaston polarizer produces separate images of the bright and dark fringes onto detector arrays. For optical systems that preserve the polarization of the scattered radiation, the first polarizer can be removed. Alternatively, the light can be additionally processed, for example, by another interferometer targeting different delay.

birefringent crystal plate of thickness $L$ and birefringence $B$ is $\tau_{0}=L B / c$. For radiation of centre frequency $v_{0}$, the interferometer signal at either of the final polarizer ports is given by

$$
S_{ \pm}=\frac{I_{0}}{2}\left(1 \pm \zeta \cos \phi_{0}\right),
$$

where $I_{0}$ is the brightness of Thomson scattered light transmitted by the first polarizing beamsplitter, $\phi_{0}=2 \pi \nu_{0} \tau_{0}=2 \pi N$ is the monochromatic birefringent phase delay and $N$ is the order of interference. The fringe visibility (fringe amplitude normalized to mean intensity) $\zeta=\zeta_{I} \zeta_{T}$ includes an instrumental component $\zeta_{I}$ due to the average of the birefringent plate delay over the angular extent of the source (analogous to the familiar slit function for grating spectrometers) as well as the degradation $\zeta_{T}$ due to the finite source spectral width. The instrument function is determined via a suitable calibration procedure as discussed in section 3 .

For a low temperature thermal distribution, the fringe visibility associated with the Thomson scattered light takes the simple form

$$
\zeta_{T}\left(\phi_{0}\right)=\exp \left[-\hat{\phi}_{0}^{2} \sin ^{2}(\theta / 2) v_{\mathrm{th}}^{2}\right]=\exp \left(-T_{\mathrm{e}} / T_{C}\right),
$$

where $T_{C}$ is a 'characteristic temperature' set by the waveplate delay and the scattering angle

$$
k T_{C}=\frac{1}{2} m_{\mathrm{e}} c^{2} /\left[\hat{\phi}_{0} \sin (\theta / 2)\right]^{2} .
$$

Optimum sensitivity to temperature variations is obtained when the optical delay is chosen such that $T_{\mathrm{e}} \sim T_{C}$, or $\Delta v / \nu_{0} \sim 1 / N$, where $\Delta v / \nu_{0}$ is the Thomson spectral bandwidth [6].

Thomson spectra and their associated interferograms for temperatures in the range $100-500 \mathrm{eV}$ for a birefringent filter based on a quartz waveplate are shown in figure 2 . The interferogram calculations take into account the wavelength-dependence of the quartz birefringence and assume that the full spectrum is observed. It can be seen that the variation of fringe visibility with temperature is best measured by setting $\phi_{0} / 2 \pi=M / 2$ where $M$ represents an integer number of half waves. At this delay, the scattered signals are

$$
S_{ \pm}=\frac{I_{0}}{2}\left[1 \pm \zeta_{T}\right],
$$

where $\zeta_{T}\left(\phi_{0}\right)$ is the Thomson spectrum fringe contrast at delay $\phi_{0}=M \pi$. The signals derived from both the orthogonally polarized light components produced by the final Wollaston polarizer are sufficient to determine the total scattered power and the contrast degradation due to the Doppler broadening:

$$
\zeta_{T}=\frac{S_{+}-S_{-}}{S_{+}+S_{-}} .
$$



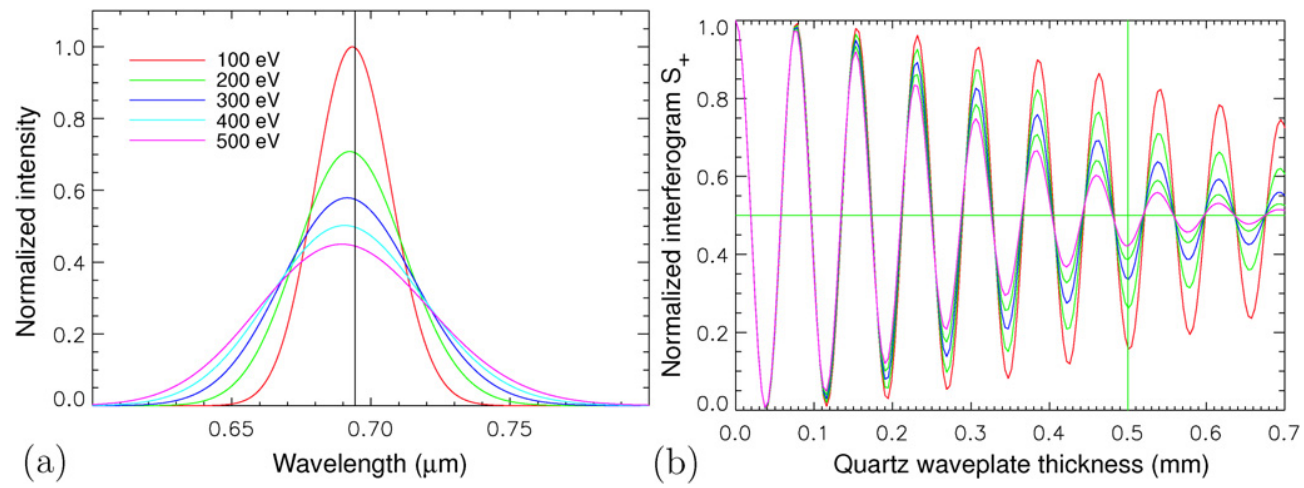

Figure 2. (a) Thomson scattered spectra for temperatures in the range $100-500 \mathrm{eV}$. We have assumed $90^{\circ}$ viewing geometry and a ruby laser source $(694.3 \mathrm{~nm})$. (b) The calculated interferograms $S_{+}$. A quartz waveplate of thickness $0.5 \mathrm{~mm}$ is optimum for sensing temperature variations near $200 \mathrm{eV}$. The calculations use the full relativistic form of the scattered spectrum and include material dispersive properties.

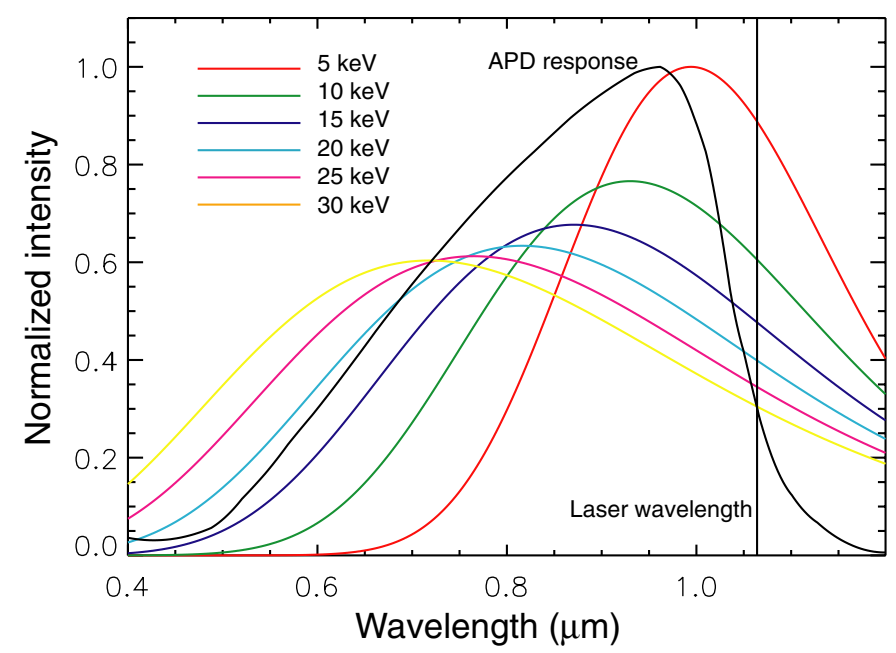

Figure 3. The Thomson spectra in the range 5 to $30 \mathrm{keV}$ for $1.06 \mu \mathrm{m}$ excitation and $90^{\circ}$ observation angle. The typical spectral response for an avalanche photodiode is superimposed for comparison.

The effects of stray light contamination are considered below.

As a concrete example, for an electron temperature $T_{\mathrm{e}}=200 \mathrm{eV}$, and $90^{\circ}$ scattering, the condition $T_{\mathrm{e}}=T_{C}$ requires $N=6.45$ waves delay at the ruby laser wavelength $694.3 \mathrm{~nm}$. $M=13$ half waves can be obtained using a crystal quartz birefringent plate of thickness $L=0.50 \mathrm{~mm}\left(n_{E 0}=1.550, n_{O}=1.541, \kappa=1.24\right)$. Because the required quartz plate is thin, sensitivity to ambient temperature changes can be ignored.

\subsection{High temperature case}

When the temperature exceeds $1 \mathrm{keV}$, the Thomson spectrum broadens and shifts to the blue. However, it is the shift of the spectrum shown in figure 3 that most sensitively conveys the temperature, and this is registered by the interferogram as a phase change. 

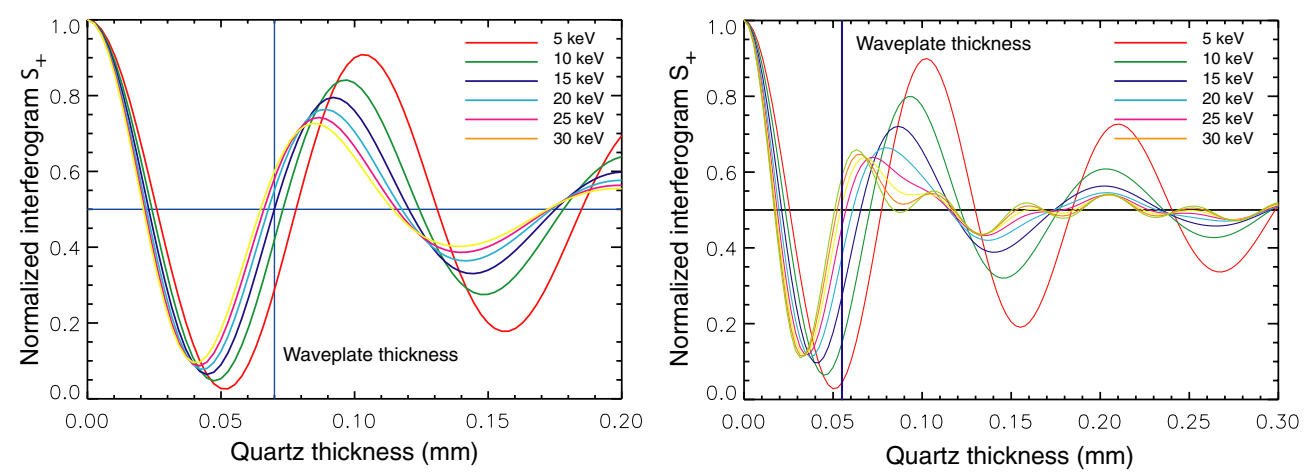

Figure 4. Interferograms $S_{+}$calculated for a quartz-based polarization interferometer in the high temperature case. (a) Interferogram for an optical system whose spectral response is determined by the APD detector element. The vertical line indicates the selected quartz waveplate thickness of $0.07 \mathrm{~mm}$. (b) Interferogram for a top-hat filter spanning $450 \mathrm{~nm}$ to $1050 \mathrm{~nm}$. The vertical line indicates the selected quartz waveplate thickness of $0.055 \mathrm{~mm}$.

To illustrate this, we model a YAG-laser based $(1.06 \mu \mathrm{m}) 90^{\circ}$ scattering system for two cases: $(a)$ an optical system dominated by the spectral response appropriate for an avalanche photodiode (i.e. no filter - see figure 3 ) and $(b)$ an ideal flat response across the range $450-1050 \mathrm{~nm}$. The calculated interferograms are shown in figures 4(a) and $(b)$, respectively.

Compared with the more narrowband case, the wideband interferograms have smaller coherence length, and, as expected, show a stronger dispersion of interferometric phase with temperature. In each case, one monitors the interferogram at a waveplate thickness selected to deliver a sensitive variation of normalized interferogram amplitude with electron temperature. The dependence of fringe amplitude on electron temperature for both the narrow and broadband cases at waveplate thicknesses $0.07 \mathrm{~mm}$ and $0.055 \mathrm{~mm}$, respectively, is shown in figure 5. It is evident that the wideband measurements are more responsive to higher temperature variations. Notwithstanding the issue of detector spectral response, the use of such large passbands also admits disproportionately more plasma continuum and line radiation background. This light will contribute to the interferogram, and must be measured separately from the Thomson pulse. To alleviate this problem, it may be desirable to observe the more compact Thomson spectrum obtained by collecting the scattered radiation at smaller angles to the probing beam.

In the case of high temperature scattered spectra, the small optical coherence length requires the use low birefringence crystals for the waveplate construction. Apart from quartz, there are a number of other crystalline materials such as lithium tantalate, $\mathrm{LiTaO}_{3}$, and magnesium fluoride, $\mathrm{MgF}_{2}$, that are suitable as delay plates. In all cases, the effects of birefringence dispersion need to be properly accounted (see (4)). Unlike $\mathrm{MgF}_{2}, \mathrm{LiTaO}_{3}$ exhibits high dispersion and the optical transmission deteriorates below $450 \mathrm{~nm}$ (figure 7(a)). Because the waveplates are thin, the poorer transmission at shorter wavelengths can be largely ignored. Nevertheless, as shown in figure 6, the stronger birefringence dispersion for $\mathrm{LiTaO}_{3}$ increases the variation of interferogram phase shift with temperature. This is countered, however, by an associated decrease in fringe visibility, with the result that the overall variation of normalized fringe amplitude $\zeta_{T}$ with temperature at high temperatures is comparable for both waveplates (figure $7(b)$ ). Also note that some ambiguity in interpretation of the fringe contrast can occur when the interferograms cross as seen in figure $7(a)$ and its associated contrast curve in figure $7(b)$. This problem can be resolved either by reducing the optical passband or choosing a different optical delay. Accurate Sellmeier equations describing the wavelength- 


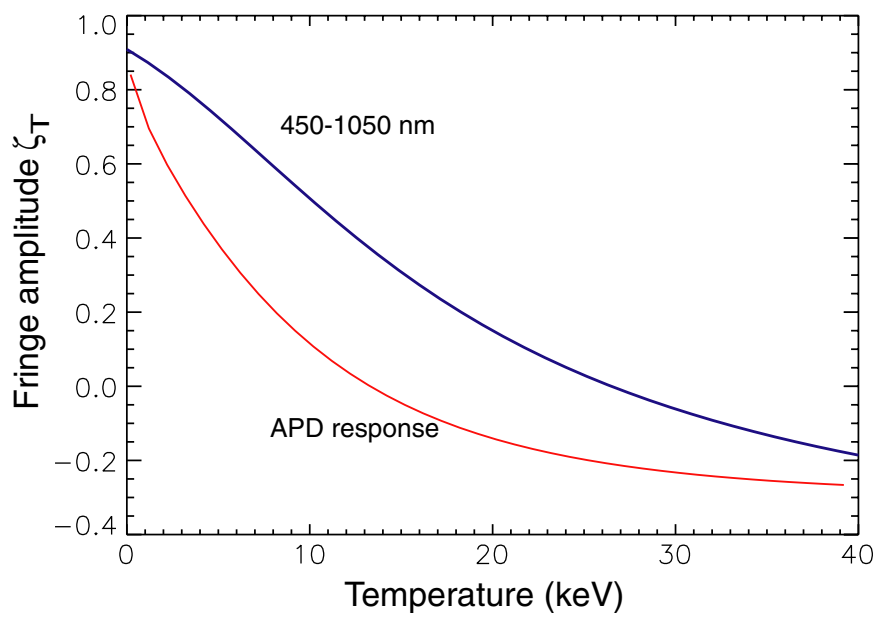

Figure 5. Variation of fringe contrast $\zeta_{T}$ with temperature for broadband systems having $(a)$ avalanche-photodiode-type response and $(b)$ ideal top-hat $(600 \mathrm{~nm})$ spectral response and for quartz waveplate thicknesses of $0.07 \mathrm{~mm}$ and $0.055 \mathrm{~mm}$, respectively. Sensitivity to high temperature variations increases with colour filter bandwidth. See text for discussion.
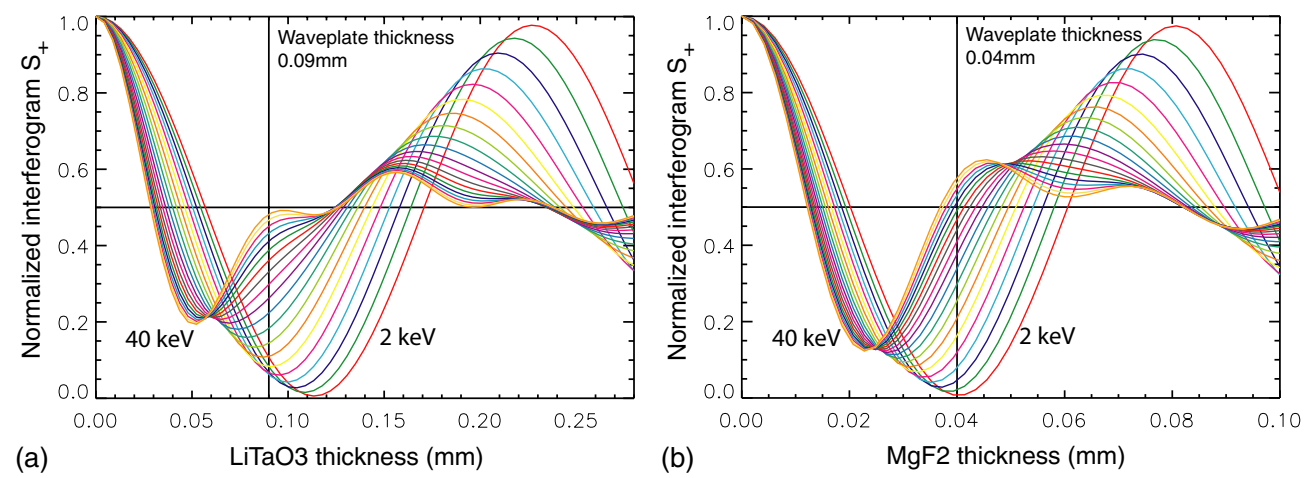

Figure 6. The Thomson interferograms in the range 2, (2), $40 \mathrm{keV}$ for $1.06 \mu \mathrm{m}$ excitation, filter passband 400-1000 nm and $90^{\circ}$ observation angle. (a) Dispersive $\mathrm{LiTaO}_{3}$ waveplate and $(b)$ $\mathrm{MgF}_{2}$ waveplate. The vertical lines in each case indicate the selected waveplate thicknesses for computation of the temperature-dependent fringe contrast.

dependence of the birefringence for the waveplate materials suggested here are readily available from manufacturers. These properties are also easily enough estimated by measuring the polarization interferometer response to light from a tunable quasi-monochromatic source.

\subsection{Modulated single-detector systems}

For multi-pulse Thomson systems, a temporal multiplex approach can be used to obtain a single-detector estimate of the electron temperature and density. A fast switching ferro-electric liquid crystal (FLC) placed between parallel polarizers, and synchronized with the laser pulse rate, can alternately switch the optical delay between zero and some fixed offset delay provided by the FLC itself. With the FLC open (fast axis parallel with polarizers, zero nett delay), the transmitted signal is $S_{+}=I_{0}$ where $I_{0}$ is the total scattered intensity. With the FLC switched 

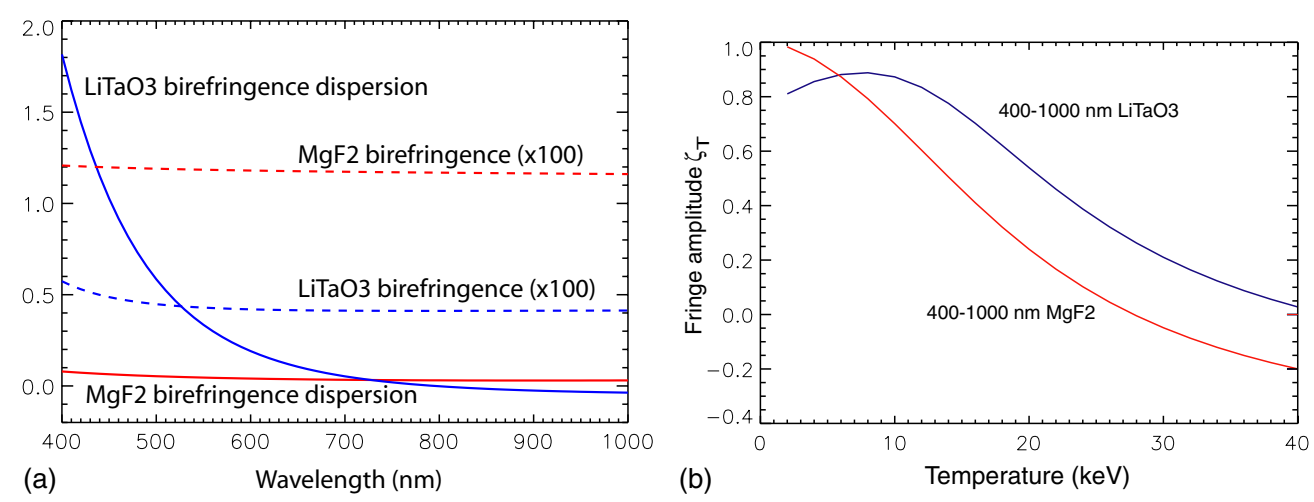

Figure 7. (a) Birefringence $B=n_{e}-n_{o}$ and birefringence dispersion $|(\lambda / B)(\mathrm{d} B / \mathrm{d} \lambda)|$ for $\mathrm{MgF}_{2}$ and $\mathrm{LiTaO}_{3}$ waveplates in the range $400-1000 \mathrm{~nm}$ and $(b)$ the variation of fringe contrast (normalized amplitude) $\zeta_{T}$ with electron temperature for ideal wide-band (400-1000 nm) filter and both $\mathrm{MgF}_{2}$ and $\mathrm{LiTaO}_{3}$ waveplates. See text for discussion.

(fast axis $45^{\circ}$ to polarizers), the signal is $S_{+}=I_{0}\left(1+\zeta_{T}\right) / 2$, where $\zeta_{T}$ is the normalized fringe amplitude at the inserted delay. The two measurements are sufficient to allow determination of the electron density and temperature provided that these quantities do not change significantly on the timescale of the laser pulse period. Interpolation techniques could be used to refine the temperature estimate when the density varies smoothly and sufficiently slowly in time.

\section{Experimental considerations}

The interferogram depends on both the nature of the scattered spectrum $I(v)$ as well as the overall system complex spectral response $R(v)$ which includes the angular dependence of the time delay (generally a small effect) as well as the spectral response of the collection and processing optics and detection system. In this case we write the normalized fringe amplitude as $\zeta=\Re(\tilde{\gamma})$ and recast equation (2) as

$$
\tilde{\gamma}=\frac{1}{I_{0}} \int_{0}^{\infty} R(v) I(v) d \nu .
$$

One means to determine the unknown response function $R(v)$ would be to measure the variation of the normalized fringe amplitude $\zeta^{\mathrm{BB}}=\Re\left(\tilde{\gamma}^{\mathrm{BB}}\right)$ for blackbody radiation as a function of the source temperature. For a given temperature $T_{j}$, (15) can be discretized in terms of the unknown spectral response coefficients $R_{i} \equiv R\left(v_{i}\right)$ as

$$
\zeta^{\mathrm{BB}}\left(T_{j}\right)=\sum_{i} I_{j i}^{\mathrm{BB}} R_{i}
$$

where $I_{j i}^{\mathrm{BB}}$ is the blackbody spectral irradiance at temperature $T_{j}$ and optical frequency $v_{i}$. For a sufficient number of measurements, (16) can be inverted for the response vector $\left\{R_{i}\right\}$. We have not attempted to estimate the condition of this procedure. Nevertheless, simulations for the case shown in figure 4 show that substantial contrast variations are achievable for blackbody temperature variations in the range 500 to $3000 \mathrm{~K}$. An alternative calibration approach is to measure the interferogram and thereby estimate the coefficients $\left\{R_{i}\right\}$ using a light source of known spectrum and a set of optical bandpass filters. With the spectral response determined, the absolute sensitivity can be obtained using either the standard Rayleigh or Raman scattering techniques [12]. 
Because of the need for the first polarizing element, the maximum throughput is only $50 \%$. However, because of the simplicity of the optical arrangement, it may be possible to install the polarization interferometer so as to view directly the polarized scattered laser light. The final polarized coherence-images of the laser stripe can then be coupled to optical fibre bundles for transport to remote detection systems.

Because the delay is small, birefringent filters will generally have a wide field-of-view and hence high throughput. Standard field-widening techniques [13] can be utilized in the case where the response is otherwise degraded by angular averaging over a range of phase delays. Numerical calculations in the low temperature case $(<1 \mathrm{keV})$ indicate negligible instrumental contrast degradation for full viewing angles up to $45^{\circ}$. In any case, off-axis or wide-field effects will be captured by the system calibration.

It is assumed that a notch or bandpass filter may be required to reduce stray light to manageable levels. In that case, the residual level of stray laser light contamination can be estimated by measuring the signals $S_{+}$and $S_{-}$in the absence of plasma. Plasma background light must also be monitored and subtracted from the measured signals $S_{ \pm}$. The unknown plasma background interferogram can be measured in a time window displaced from the laser pulse and then subtracted from the overall signals. Simulations show that, in the presence of background plasma light and its attendant shot noise, the temperature estimate for both the birefringent filter and dual interference filter cases is biased whenever the scattered intensities are unbalanced. For the birefringent filter, this effect can be minimized by targeting the interferometric optical delay so as to approximately balance the bright and dark fringe intensities for the expected range of electron temperatures.

In general the problems associated with a high plasma background are common to both interference and birefringent filter systems. Nevertheless, at high plasma temperatures, plasma background bremsstrahlung may become problematic, and this would require an increase in the low pass-cutoff wavelength, thereby compromising the temperature resolution at high temperatures. While increased laser pulse energy would alleviate this problem, another solution might be to combine the use of a modulated high power continuous-wave laser with a wide-band birefringent filter and synchronous detection techniques.

Instrument issues relating to optical construction, field-widening, calibration and the effects of background radiation and noise will be considered more fully in a later paper.

\section{Conclusion}

The optical systems proposed here offer some advantages over traditional polychromator systems for the analysis of Thomson scattering spectra. Birefringent filters can have high transparency, large apertures and a wide, uniform field-of-view. They are therefore ideal for 2D time-resolved wideband spectral imaging applications. The Thomson systems described here utilize a single filter and one or two detector arrays to obtain images of the electron temperature and density.

\section{Acknowledgments}

I would like to thank Dr T. Hatae of JT-60U for useful discussions and for providing information relating to the nature of the high temperature Thomson spectrum. I am also indebted to the referees whose suggestions have improved this paper. 


\section{References}

[1] Sheffield J 1975 Plasma Scattering of Electromagnetic Radiation (New York: Academic Press)

[2] Beurskens M N A, Barth C J, Lopes Cardozo N J and Van der Meiden H J 1999 Plasma Phys. Control. Fusion 411321

[3] Dimock D et al 1997 Rev. Sci. Instrum. 68700

[4] Yoshida $\mathrm{H}$ et al 1999 Rev. Sci. Instrum. 70747

[5] Howard J, Michael C, Glass F, and Cheetham A 2001 Rev. Sci. Instrum. 72888

[6] Howard J 2002 Appl. Opt. 41197

[7] Howard J, Michael C, Glass F, and Danielsson A 2003 Plasma Phys. Control Fusion 451143

[8] Hsieh C L, Bray B D and Liu C 2004 Rev. Sci. Instrum. 753897

[9] Born M and Wolf E 1980 Principles of Optics (Pergamon, Oxford)

[10] Naito O, Yoshida H and Matoba T 1993 Phys. Fluids B 54256

[11] Hutchinson I H 1987 Principles of Plasma Diagnostics (Cambridge: Cambridge University Press) p 218

[12] Howard J, James B, and Smith W 1979 J. Phys. D: Appl. Phys. 1435

[13] Steel W 1967 Interferometry 1967 (Cambridge: Cambridge University Press) 